\title{
Polysèmes
}

Revue d'études intertextuelles et intermédiales

11 | 2011

Cadres

\section{Le cadre entre le champ et la figure}

\section{Marie-Madeleine Martinet}

\section{(2) OpenEdition}

\section{Journals}

Édition électronique

URL : http://journals.openedition.org/polysemes/657

DOI : $10.4000 /$ polysemes.657

ISSN : 2496-4212

Éditeur

SAIT

Édition imprimée

Date de publication : 1 janvier 2011

Pagination : 263-277

ISSN : 0999-4203

\section{Référence électronique}

Marie-Madeleine Martinet, «Le cadre entre le champ et la figure », Polysèmes [En ligne], 11 | 2011, mis en ligne le 01 mars 2015, consulté le 30 avril 2019. URL : http://journals.openedition.org/ polysemes/657 ; DOI : 10.4000/polysemes.657

Ce document a été généré automatiquement le 30 avril 2019.

Polysèmes 


\title{
Le cadre entre le champ et la figure
}

\author{
Marie-Madeleine Martinet
}

1 Le motif du cadre, élément structural, rayonne sur des notions comme l'arrière-plan, ou les rapports d'ombre et lumière ; par ce biais, il articule les relations d'image et celles de contiguïté, celles de champ et de figure, pour aboutir à ouvrir sur l'irréel.

\section{Cadrage et quadrillage}

2 Les peintres utilisent souvent des effets de cadre intérieur: porte, rideau, au-delà desquels on voit une scène. Cet effet simple dans lequel un élément est cadrant et les autres encadrés peut conduire à des cas plus compliqués avec superposition des rôles. Ainsi dans une vue à travers une colonnade, les entrecolonnements situés en face du regard du spectateur s'ouvrent sur le lointain et servent de cadre intérieur, mais pour ceux qui sont à l'oblique à l'extrémité de la colonnade, les colonnes se superposent dans le regard du spectateur et ne laissent plus passer le regard au-delà : elles sont encadrées par le regard et non plus cadrantes.

3 On voit cet effet dans une vue de Covent Garden par Thomas Malton (1775), où, au milieu et du côté gauche, nous apercevons entre les piliers les rangées de maisons situées de l'autre côté, tandis qu'au fond à droite nous ne voyons que les piliers. 


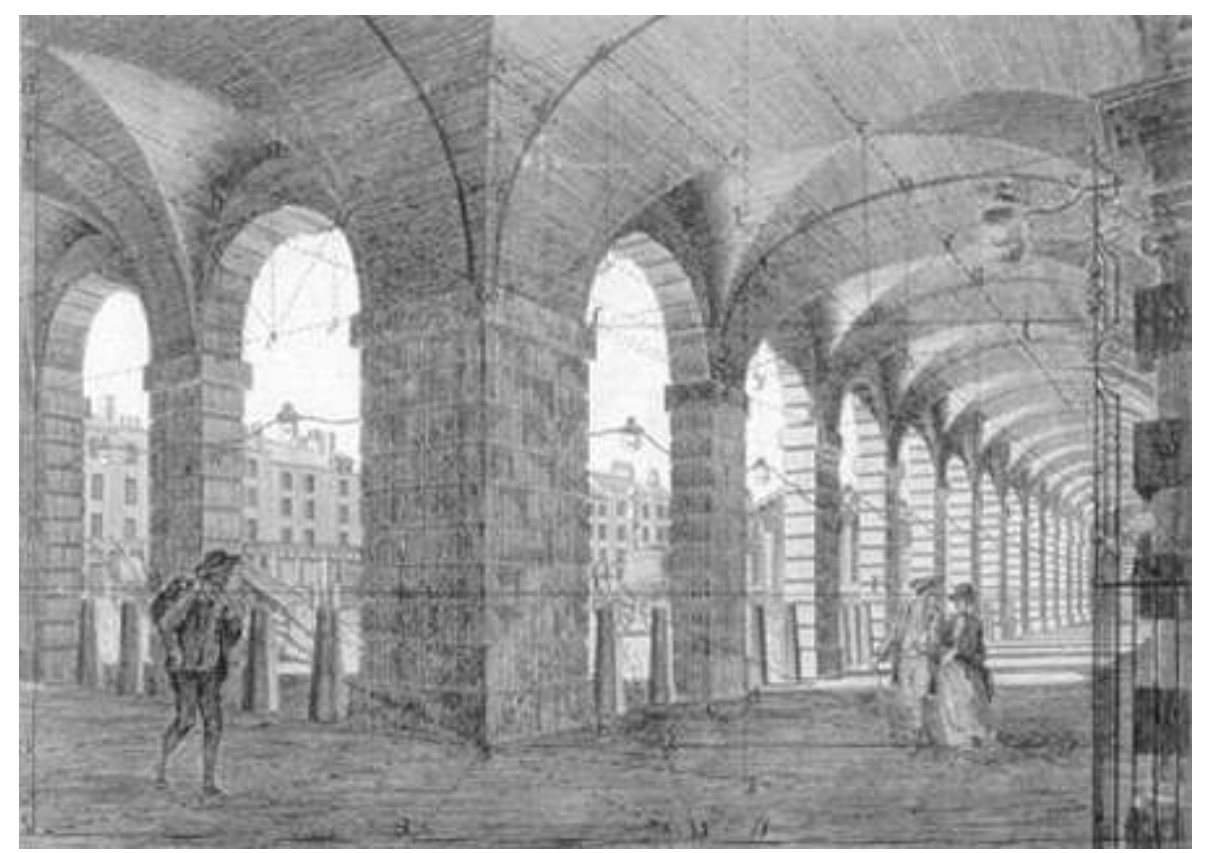

Thomas Malton, Covent Garden, Studies in Perspective Construction (1775)

http://www.18thc-cities.paris-sorbonne.fr/Perspective\#6

L'explication est simple :

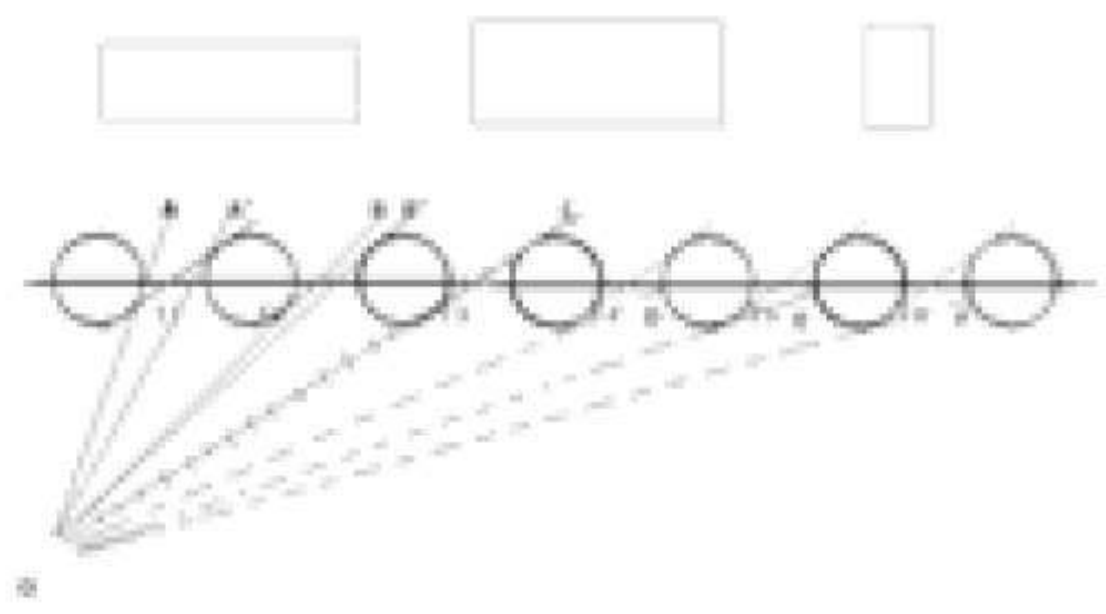

Un personnage dont le regard est situé en $O$ verra les bâtiments derrière la colonnade par les entrecolonnements $\mathrm{AA}^{\prime}$ et $\mathrm{BB}$ ', mais pas pour les parties plus à droite. On trace entre les colonnes des obliques passant entre les colonnes de façon à être tangentes à l'une d'un côté de l'axe et à l'autre de l'autre côté (ici en bleu, T1, T2 etc) : ces tangentes font avec l'axe de la colonnade un angle déterminé identique tout du long. Tant que le rayon visuel du spectateur (en rouge) est presque perpendiculaire à la colonnade, et qu'il fait avec l'axe un angle plus grand que cet angle des tangentes, comme en OA-OA' et en OB-OB', le regard passe entre les colonnes; en C; le rayon visuel coïncide avec la tangente et le spectateur voit les deux colonnes juxtaposées (ligne avec croix); ensuite, quand le regard devient plus oblique (lignes en tireté), il bute sur les colonnes en D, E, F.

Il s'ensuit, dans l'imagination du spectateur, un double motif; la vue est celle d'un faisceau d'obliques rayonnant à partir du point de vue, alors que le plan imaginé du square est fait d'un quadrillage de parallèles : 


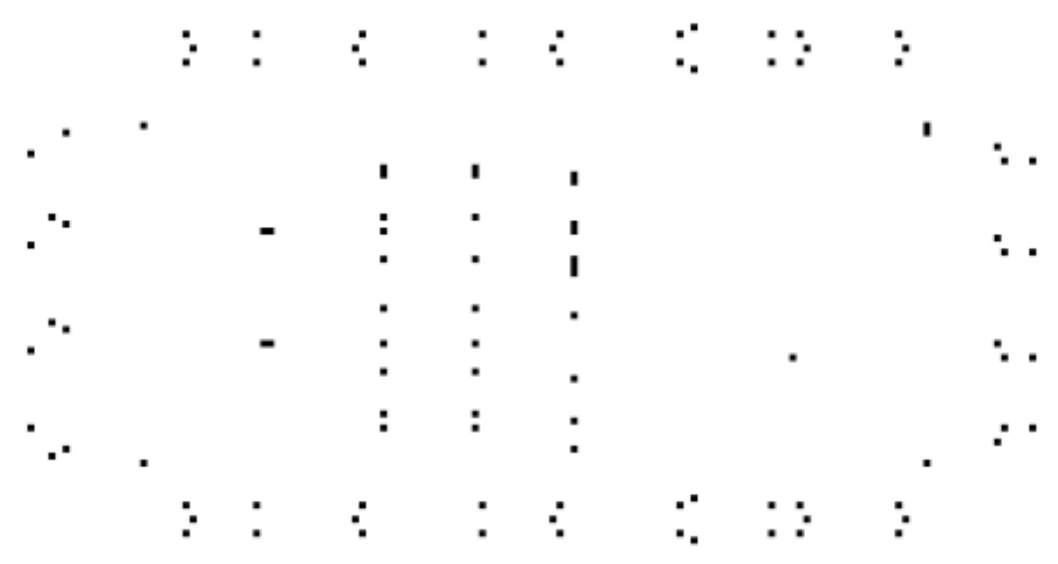

Retournons à la gravure de Malton: c'est au point où les colonnes cessent d'être cadrantes pour devenir cadrées qu'il a placé les promeneurs, là où les colonnes cessent d'être la projection du regard du sujet pour devenir l'objet, que se trouvent des observateurs symétriques de nous.

En un contraste souligné par Diderot, le cadrage, cet artifice, s'il vient du regard du spectateur plus que de l'objet regardé, peut donner une illusion de réel plus grand. Il explique à propos de Joseph Vernet que si on regarde un tableau avec une lunette, dont le cadre de vision se substitue au rebord du tableau, on se croit dans la réalité :

Regardez le Port de La Rochelle, avec une lunette qui embrasse le champ du tableau, et qui exclut la bordure; et oubliant tout à coup que vous examinez un morceau de peinture, vous vous écrierez, comme si vous étiez placé au haut d'une montagne, spectateur de la nature même : O le beau point de vue $!^{1}$

Ainsi le cadrage est un lieu d'articulation entre la vue subjective et la vue objective.

\section{La réintégration du motif dans un arrière-plan comme source d'inspiration}

7 L'imagination des artistes se développe à partir d'un motif central qui se replace dans plusieurs arrière-plans. Le cadrage devient ainsi l'ensemble de l'arrière-plan. Ainsi on retrouve chez Canaletto une vision d'un dôme au-dessus de l'eau, souvenir de sa Venise de jeunesse avec la Salute dominant l'embouchure du Grand Canal, devenu dans ses tableaux londoniens le dôme de Saint-Paul replacé sur un nouvel arrière-plan, celui de la Tamise - par exemple la veduta vénitienne de 1730 (National Gallery), et la vue de Londres de 1747 (Goodwood House). De nos jours, les animations sur support numérique nous permettent de faire apparaitre un motif constant de dôme qui se réintègre en fondu dans plusieurs arrière-plans successifs, Venise et Londres ${ }^{2}$.

\section{Ombre, image et contiguïté}

En plus des cadrages évidents, certains motifs peuvent en tenir le rôle. L'ombre forme-telle un cadre partiel pour son objet? C'est une question visuelle, une zone d'ombre entourant l'objet pouvant être comme un motif de cadre intérieur autour de lui, et c'est aussi une question philosophique, car du XVIII ${ }^{e}$ siècle aux sciences cognitives actuelles on s'interroge sur le rôle de l'ombre dans la perception et dans l'identification des objets. 
9 L'ombre a un rôle structural, celui de cadrer les objets principaux tout en conférant au tableau des lignes de composition intérieures. Dans ce même tableau de Canaletto, les ombres, qui vont à l'inverse de l'oblique de la Tamise comme motif structural, servent aussi à souligner les motifs principaux, surtout le dôme, et aussi les quais, les bateaux, les personnages.

10 Mais les ombres donnent en fait une variante inversée des effets de cadrage: contrairement au cadre qui préexiste à l'objet qui s'y insère, elle est dépendante de lui ; elle est aussi dépendante du temps qu'elle introduit dans le tableau. Enfin, alors que le cadre est généralement différent de ce qu'il encadre, l'ombre est une quasi-image (plus ou moins déformée) de son objet. Ainsi elle combine image et contiguïté, métaphore et métonymie.

11 Les effets de cadrage intérieur sont en fait une superposition ou une articulation de fonctions. Le cadre, comme l'ombre qui est un de ses cas particuliers, a la double fonction de similarité et de contiguïté, comme l'explique une étude comparée des trois auteurs majeurs sur ce sujet, Gombrich, Baxandall et Stoichita :

Shadow enjoys a special status - shared with reflection - in the tropical cocktail. The tropes of metaphor and metonymy are determined by, respectively, likeness and contiguity. Almost all signs work by means of one or the other. We could introduce Peirce's distinctions also, and speak of the index as metonymic, of the icon as metaphoric. What we notice about shadows and reflections - and it is surely this that gives them such literary prestige - is that they combine both likeness and contiguity. They only exist in contiguity with that which causes them. At the same time, as likenesses they function as images of what need not or (in the case of self) cannot be seen. A shadow is, indexically, a pointer to that which is its cause, as well as being an icon, in outline, of its cause. ${ }^{3}$

12 Ainsi, pour montrer comment la mise en abyme (similarité) interfère avec les jeux d'arrière-plan et de premier plan (contiguïté), on a donné l'exemple de l'ombre d'une danseuse projetée sur la scène, qui peut être vue en plus comme un arrière-plan ou un premier plan :

The projected image can be perceived either as a visual "mise en abyme", a dynamic background (or foreground when a scrim is used), a novel light source or even as an actor, depending on the given artistic means, uses, and contexts. ${ }^{4}$

\section{Ombres et cadres comme ouverture sur les espaces irréels}

13 De nombreux travaux de sciences cognitives ont étudié l'ombre en relation avec le contour et avec le vide (méréologie, notamment recherches de Roberto Casati). L'ombre interfère avec le cadrage par le biais du contour; en effet si on trace des traits sur une ombre, y compris son propre contour, elle cesse d'être vue comme ombre pour être perçue comme un objet indépendant. Ainsi, ajouter un cadre à l'ombre fait qu'elle cesse d'être cadre de sa source ${ }^{5}$. Les modes de perception des contours, et surtout d'interpolation des contours absents, font apparaître les modes du jeu entre le réel et l'irréel, lignes virtuelles imaginées entre des points, et inversement lignes amodales pour l'interpolation des contours cachés ${ }^{6}$.

L'ombre est aussi une articulation entre des espaces contenus les uns dans les autres, encadrés les uns par les autres, les espaces de dimensions supérieures ou hyperespaces. Puisqu'une ombre d'un objet en 3 D est elle-même en 2 D, inversement on peut déduire un 
objet de dimension supérieure à partir d'une ombre, une ombre en $2 \mathrm{D}$ suggérant la présence d'un objet en $3 \mathrm{D}$, et l'on peut poursuivre au-delà des limites de notre perception : s'il existe une ombre en $3 \mathrm{D}$, elle suppose un objet en $4 \mathrm{D}^{7}$.

La réflexion sur les espaces de dimensions supérieures a mené à l'idée du cadrage comme lisière entre l'intérieur et l'extérieur. Dans le Flatland d'Abbott (1884), les personnages qui vivent en deux dimensions dans le plan ne peuvent voir leur maison d'en haut comme nous le faisons, ils ne peuvent avoir l'idée d'intériorité de leur maison alors que nous voyons un plan qui nous montre les espaces intérieurs; pour connaître l'intériorité, il faut passer à une dimension supérieure.

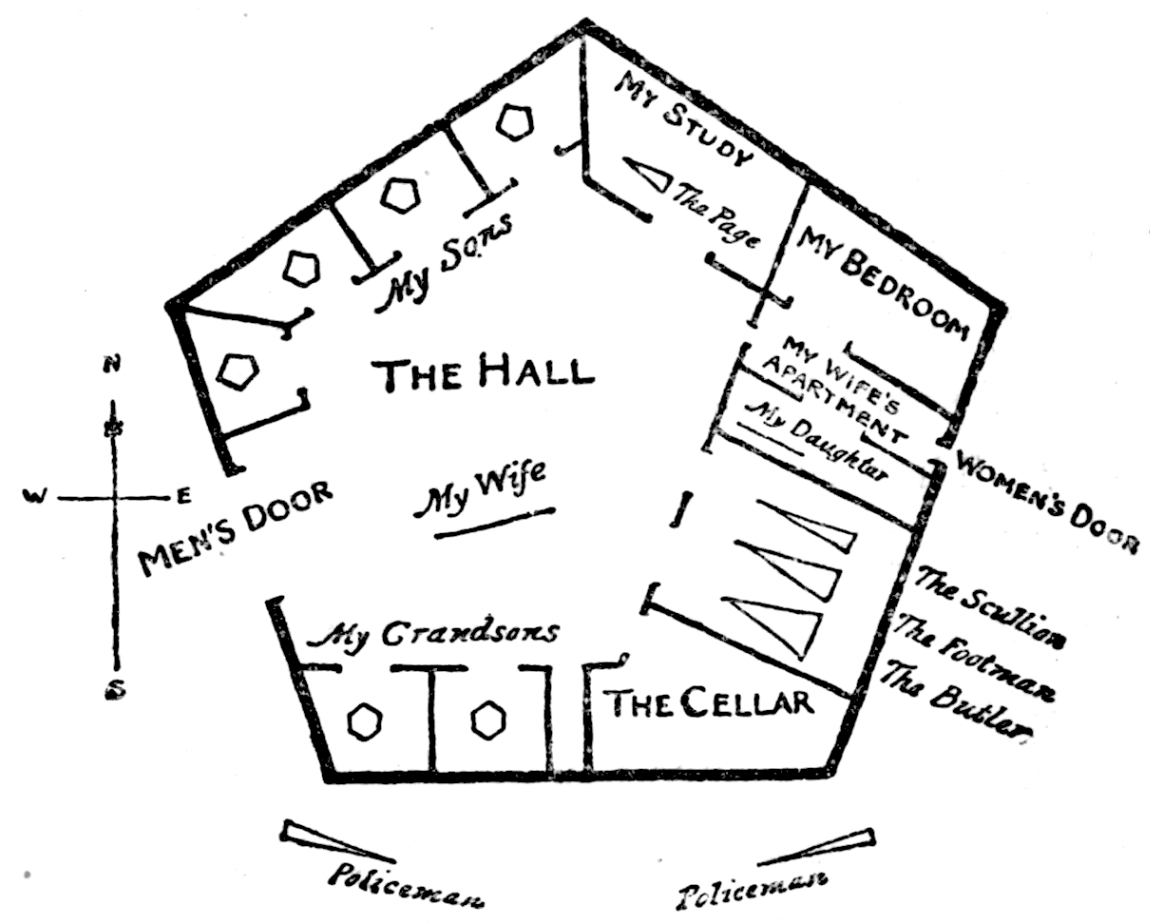

Edwin Abbott, Flatland, a Romance of Many Dimensions, London: Seeley and Co.,1884, 79, Creative Commons

\section{Contours et atmosphère environnante}

Si l'ombre oscille entre l'intérieur et l'hyperespace, une question se pose pour la lumière environnante : est-elle le reflet de son objet ou son enveloppement?

Hogarth, dans The Analysis of Beauty (1753), étudie comment nous cernons les objets, l'espace encadré dans un objet et l'atmosphère extérieure.

Il traite un objet comme un espace intérieur enclos dans les contours, donc sujet à la variété comme l'extérieur :

([I]f a solid figure be the subject of the composition) the contents or space that is to be inclosed within those lines, must be duly consider'd and var'd too, ... [St Paul's]: the noble projecting quantity of a certain number of them, which presents bold and distinct parts at a distance, when the lesser parts within them disappear; and the grand few, but remarkably well-varied parts that continue to please the eye as long as the object is discernable. (Ch.VIII) 
Il y voit ainsi une fonction de la distance: les cadrages intérieurs dépendent de l'observateur.

Pour l'espace extérieur, l'atmosphère forme-t-elle un cadrage, joue-t-elle un rôle alors qu'on pourrait ne pas y prêter attention? Hogarth attire plusieurs fois l'attention sur elle comme élément important du tableau; il prend des caractéristiques techniques de certaines formes de gravure comme traits fondamentaux de la vision spatiale, et dans son chapitre sur le procédé de la manière noire, il considère le fond comme primordial et les figures comme secondaires, en une inversion, et selon une analogie avec les effets atmosphériques :

Of L I G H T and SH A D E, and the manner in which objects are explained to the eye by them.

Mezzo-tint: I have often thought that a landskip, in the process of this way of representing it, doth a little resemble the first coming on of day. The copper-plate it is done upon, when the artist first takes it into hand, is wrought all over with an edg'd-tool, so as to make it print one even black, like night: and his whole work after this, is merely introducing the lights into it; which he does by scraping off the rough grain according to his design, artfully smoothing it most where light is most required: but as he proceeds in burnishing the lights, and clearing up the shades, he is obliged to take off frequent impressions to prove the progress of the work, so that each proof appears like the different times of a foggy morning, till one becomes so finish'd as to be distinct and clear enough to imitate a day-light piece. (Ch.XII)

Hogarth applique des termes argumentatifs au processus de perception et d'identification des objets : les objets sont "expliqués" au regard, et les ombres jouent un grand rôle dans l'isolement de l'objet par rapport à son arrière-plan. Il prend comme exemple la gravure à la manière noire, dans laquelle les rapports entre le fond et le motif sont inversés par rapport aux autres types de dessin; on fait d'abord un fond noir en rendant rugueuse la surface entière puis on y fait apparaître les objets en clair en écrasant les rugosités : le fond a priorité. Hogarth considère que ce mode est le plus proche de la nature. En une analogie atmosphérique, il compare cette technique à l'apparition du jour au milieu d'un fond nocturne. Le médium technique devient support du rapport entre le champ et la figure.

21 Il argumente que la perception distingue les objets par contraste avec ce qui les entoure, en y ajoutant le sens de la profondeur ou relief; il faut tenir compte que nous sommes à cette époque au moment d'articulation entre les deux sens de shade, ombre et nuance, pour expliquer ce passage de la couleur au relief:

[...] separating and relieving the several objects by the different strengths or shades of them being opposed to each other. The other shades that have been before spoken of, serve and help to the like purposes when properly opposed; but as in nature they are continually fleeting and changing their appearances, either by our or their situations, they sometimes oppose and relieve, and sometimes not. (Ch.XIII) Il y a une opposition de plans, représentée par les termes relieve, oppose. Ces oppositions qui forment un cadrage de plans sont un schéma où les formes jouent presque seules, comme le montre la gravure accompagnant le texte :

Those objects which are intended most to affect the eye, and come forwardest to the view, must have large, strong, and smart oppositions, like the fore-ground in fig. $89 \mathrm{Pl}$ 2. (Ch.XIII) 


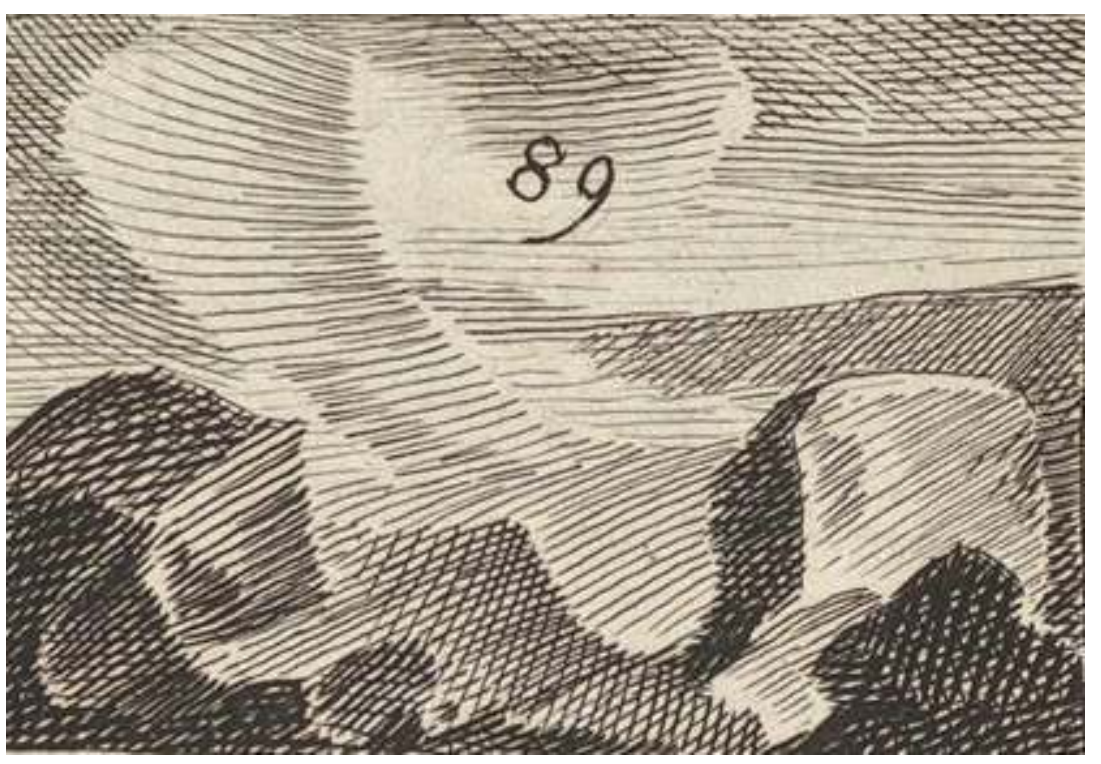

L'échange de fonctions entre éléments cadrants et éléments cadrés revient aux rapports entre le cadrage géométrique et le cadrage atmosphérique. La construction perspective, comme l'a montré Hubert Damisch, combine deux modalités d'appréhension de l'espace, la boîte cadrante quadrillée, et le repérage des points de fuite à l'infini, or le nuage est à la fois la limite d'un espace cadré et une suggestion de prolongement vers l'indéfini, associant ces deux modalités ${ }^{8}$. Ce sont les éléments flous qui jouent ces deux fonctions.

De même, un élément structural du paysage classique est le pont, étudié par Louis Marin, $\mathrm{du}$ fait que ses arches cadrent un vide: "clôture-ouverte ". Or le vide, sujet de découvertes en physique à l'époque, fut d'abord un élément encadré - découvert à l'intérieur d'une pompe - avant de devenir un environnement.

La perception du rapport cadrant / cadré a dominé les premières études sur la rémanence de la vision, au début du XIXe siècle. Les expériences sur ce phénomène ne furent pas celles des diverses positions d'un objet mouvant (un coureur, comme dans les expériences de la fin du XIXe siècle), ce fut celle de l'image reconstituée en mêlant un motif et son cadre : l'oiseau et la cage, placés chacun sur un côté de la feuille, qui se superposent quand on fait tourner la feuille et font apparaître l'oiseau dans la cage ${ }^{10}$.

26 Ces rapports entre motif et arrière-plan, plus récemment, ont été appliqués à l'interprétation des concepts et du langage : la notion de la relation « profile/base » dans le sens d'un mot (l'objet désigné, et les corollaires de sens nécessaires) relèvent de l'apparition d'un arrière-plan ou d'un champ derrière l'objet: dans le mot «île », la définition implique de faire allusion, en plus du territoire de l'île elle-même, à ce qui n'est pas l'île, la mer qui l'entoure ${ }^{11}$.

\section{Variations entre champ et figure}

Il est habituel de considérer les formes massives comme figure et les vides comme champ, mais encore l'art inverse-t-il souvent ces fonctions. Et encore, lequel est cadre et lequel est cadré ? Dans un paysage urbain, un bâtiment placé vers le centre sera identifié comme motif central et les places alentour comme vide et cadrage atmosphérique, c'est le cas le plus simple, mais si le tableau est composé comme une rue centrale flanquée de bâtiments, où est la figure et où est le champ? Si nous décidons que le vide central, la rue, 
devient la figure, et que les bâtiments alentour deviennent le champ, ils prennent la fonction de cadrage intérieur. Ainsi le paysage urbain a causé une nouvelle conception de l'espace; dans des motifs comme le crescent et le square, l'architecte voit le plein et l'urbaniste le vide. Pourrait-on dire qu'il y a cadre intérieur lorsqu'un élément habituellement figure devient élément structural du champ?

\section{Modalité et hypothèse, temps, composition} champ et de figure en espaces successivement emboîtés; or ces cadrages successifs fonctionnent sur le mode de l'hypothèse, et ils introduisent les espaces conditionnels : si sur ce paysage, qui contient champ et figure, nous ajoutons ce temple, qui devient figure, le paysage précédent avec champ et figure devenant en entier champ...

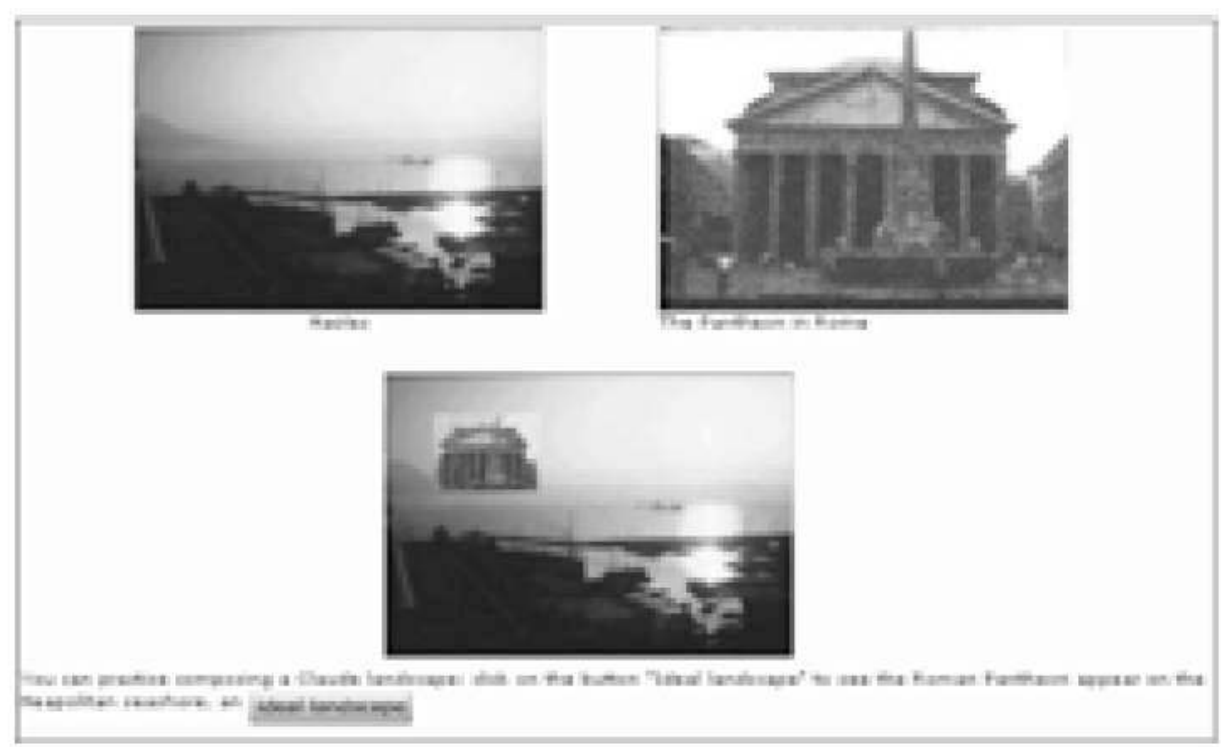
donner au nouveau temple la propriété d'être invisible ou visible selon que l'observateur choisit de l'insérer dans le paysage, le placer sur un support rectangulaire transparent nommé « calque » (layer) : c'est lui qui peut être affecté de la propriété d'alternance entre la visibilité et l'invisibilité, entraînant avec lui le temple qui y est placé. Ce qui nous fait réfléchir sur les rapports entre les objets et leur cadrage : chaque objet est en effet placé sur son propre cadrage, et ils s'emportent mutuellement. Les relations doubles de motif à paysage sont en fait ternaires : le motif, son cadrage, et le paysage cadre plus large ; ou même plus précisément à quatre éléments puisque le grand paysage lui-même est sur son propre calque.

Dans des motifs décoratifs contemporains, il est habituel d'utiliser des motifs comme cadrage dans lesquels apparait un autre motif. Ainsi le programme du Louvre, où des motifs floraux font comme une fenêtre à travers laquelle s'entraperçoit l'Odalisque. Si les motifs floraux avaient été seuls, ils auraient compté pour leur partie intérieure, qui aurait été la figure. Ici, ils sont au contraire uniquement la limite des zones extérieures qui sont le champ, et c'est l'odalisque vue de façon fragmentaire qui est la figure.

31 Ces échanges par lesquels une figure devient contour de champ sont permis, et donc favorisés, par certains instruments actuels de dessin. 


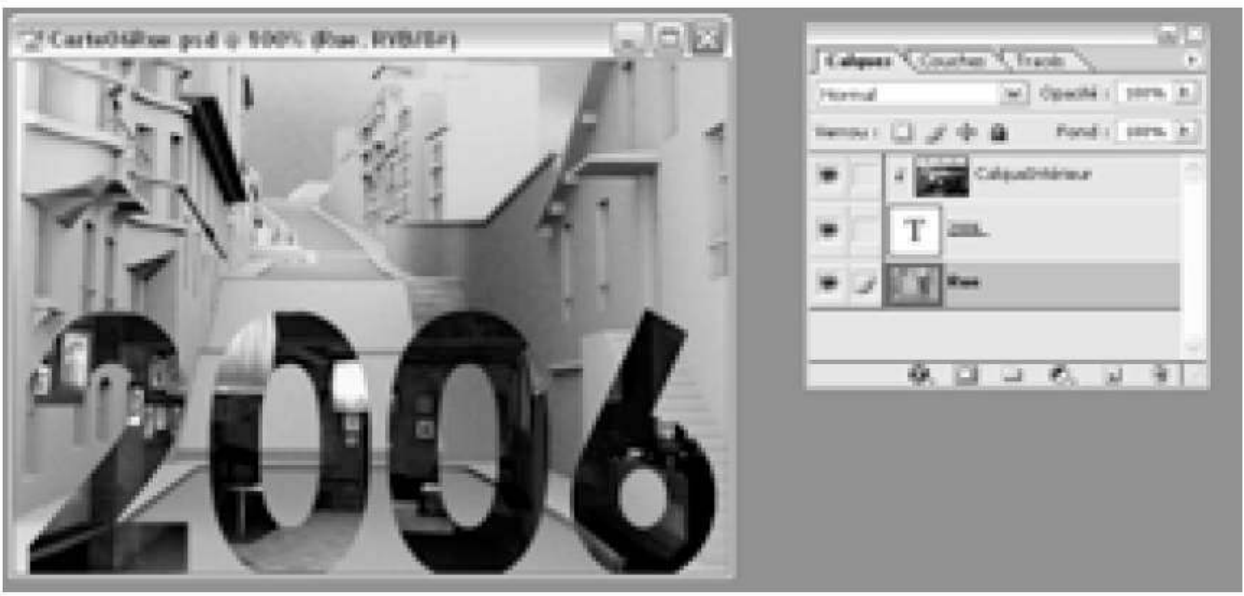

32 C'est dans les logiciels d'image la technique du «masque d'écrêtage » (clipping group). C'est, comme on le voit dans un exemple d'écran de travail faisant apparaitre la palette des « calques » (layers), une combinaison de trois éléments : l'arrière-plan, la vue aperçue à travers les lettres, et le motif de chiffres qui, jadis motif plein, sert de frontière maintenant qu'il est un vide. La petite flèche recourbée associant les deux éléments de la vue intérieure et des chiffres leur attribue cet effet de «masque d'écrêtage » faisant que la première est vue à travers les seconds.

Il a été dit que la notion de "champ » était appréciée dans la deuxième moitié du $\mathrm{XX}^{\mathrm{e}}$ siècle du fait qu'elle supprimait l'opposition entre l'art figuratif et l'art abstrait ${ }^{13}$. N'en est-il pas de même pour le cadre, qui, au lieu de se rapporter aux deux partenaires « champ / figure », se rapporte à leur lisière?

\section{NOTES}

1. Denis Diderot, «Salon de 1763 », Essais sur la peinture ; Salons de 1759, 1761, 1763 (Paris : Hermann, 1984), 228-29.

2. Voir le cdrom Georgian Cities (Paris : CATI, 2000).

3. Charles Lock, “A Returning of Shadows", Literary Research / Recherche littéraire 29 (printempsété 1998)

http://epe.lac-bac.gc.ca/100/201/300/literary_research-ef/n28-n36/old29/clock.htm perception from shapes and shadows (an article from the MIT Encyclopaedia of Cognitive Science, 1999.

4. http://www.contempaesthetics.org/newvolume/pages/article.php?articleID=235

5. http://roberto.casati.free.fr/shadowmill.com/shadowszones.htm

6. http://journalofvision.org/3/4/4/article.aspx

7. Thomas Banchoff, Beyond the Third Dimension: Geometry, Computer Graphics, and Higher Dimensions , New York: Scientific American, 1990, passim.

8. Hubert Damisch, Théorie du nuage : pour une histoire de la peinture, Paris : Seuil, 1972, 226.

9. Louis Marin, "Pour une sémiologie picturale" (1968), Études sémiologiques, écritures, peintures, Paris : Klincksieck, 1971, 27.

10. http://brightbytes.com/collection/thaum.html 
11. John R. Taylor, Cognitive Grammar, Oxford: OUP, 2002, 198.

12. http://www.cati.paris4.sorbonne.fr/cours/maitrise/man404/virtual_spaces/composite1.php

13. Julian Bell, What is Painting?, London: Thames and Hudson, 1999, 217.

INDEX

oeuvrecitee Analysis of Beauty (The), Flatland 